\title{
Black vs African American: Why are communication and clinical researchers not paying attention to what descendants of the African Diaspora want to be called, and why is that a problem?
}

\author{
Raiven Owusu ${ }^{1}$, Katherine Ridley-Merriweather ${ }^{2}$, Krista Hoffmann-Longtin ${ }^{1,3}$ \\ ${ }^{1}$ Indiana University School of Medicine, ${ }^{2}$ Susan G. Komen Tissue Bank at the IU Simon Cancer \\ Center, ${ }^{3}$ Indiana University Purdue University Indianapolis
}

Problem: Descendants of African Diaspora (DADs) in the U.S. experience greater health disparities than other racial or ethnic minority groups. Many factors play a role, including their lack of participation in clinical trials. By refusing to participate, DADs cannot benefit from medical research. The barriers to recruiting DADs is well discussed in literature, but we found no research suggesting that mislabeling participants could be creating another barrier. This essay delves into the history of labeling DADs to illuminate the existing tensions between the use of "Black" and "African American" and the impact they may have on recruitment.

Processes: Communication Theory of Identity and Critical Race Theory shaped our analysis of this mislabeling issue and its possible implications. Both theories offer insight into how an individual shapes and is simultaneously shaped by communication. We reviewed, summarized, and coded literature in the academic and popular press to answer the research question: How can mislabeling DADs possibly deter them from participating in clinical trials?

Findings: Within DADs, there are several subgroups with different cultural heritages that contribute to how people identify. Therefore, no "catch-all" label can be used to accurately describe DADs. Academic and popular press literature indicate that preferences exist amongst this group for how they wish to be identified. Researchers should realize that "Black" and "African American" are not perceived the same by many DADs and should refrain from using them interchangeably.

Conclusion: Given the current Black Lives Matter movement, it is imperative that health communication scholars and health researchers consider how language shapes participation and research outcomes for DADs. More research is needed to determine if the use of "Black" and "African American" interchangeably creates another barrier to the recruitment of DADs. However, if possible, researchers should take time to note the preferences of their target populations prior to recruitment. 